\title{
Prevalencia de lesiones mucosas en población anciana de la Región de Murcia
}

\author{
López Jornet P*, Saura Pérez $M^{* * *}$
}

\section{RESUMEN}

Introducción: El estudio de la prevalencia de la patología oral es importante, tanto para conocer el estado de salud como las necesidades de tratamiento. En este trabajo se valora la presencia de las lesiones mucosas orales en mayores de 65 años en pacientes institucionalizados y no institucionalizados de la Región de Murcia Pacientes y método: Se estudio una muestra representativa de ancianos de la región de Murcia igual o mayor de 65 años en total 385 sujetos; tasa de participación $46 \%$. Se diagnosticaron las lesiones mucosas en base a los criterios establecidos por la OMS

Resultados: La prevalencia de las lesiones orales fue de 17,8\% (no institucionalizado) y $14,2 \%$ institucionalizado. La lesión mas frecuente asociada fue la ulcera traumática seguida de lengua geográfica y candidiasis protésica. Conclusión: Realizar programas de prevención y diagnostico de lesiones de mucosa oral. Además en los casos que se establezcan tratarlas adecuadamente.

Palabras clave: Patología oral, edad, institucionalizados, mucosa oral.

\section{SUMMARY}

Background: Oral prevalence studies are important to know the state of health and the needs of treatment. The aim of the study was to determine the oral health status in a group of Murcia individuals 65 years of age or older Patients and Methods: A sample of 385 representative Murcia subjects older than 65 years old were (response rate $46 \%$ ) Oral mucosal lesions were gathered according World Health Organization.

Results: The prevalence of oral lesions was 17,8\% (non-institutionalised) and 14,2 (institutionalised). The most common lesion was traumatic ulcer followed geographic tongue and denture stomatitis.

Conclusions: That oral mucosal lesions are common in elderly people in Murcia, suggesting the necessity for improved standards of prevention, and diagnostic and opportune treatment of these lesions.

Key words: Oral pathology, elderly; institutionalised; oral mucosa.

Aceptado para publicación: Abril 2006.

* Prof. Titular Medicina Bucal. Universidad de Murcia.

** Prof. Asociado Periodoncia. Universidad de Murcia.

López Jornet P, Saura Pérez M. Prevalencia de lesiones mucosas en población anciana de la Región de Murcia. Av. Odontoestomatol 2006; 22 (6): 327-334.

\section{INTRODUCCIÓN}

La evolución demográfica, debido a el aumento del grupo de población mayor de 65 años, va a ser una de las principales causas de un profundo cambio de planificación desde el punto de vista social y sanitario. El conocimiento de la salud oral es esencial para poder diseñar y realizar programas de prevención. 
En el campo de salud oral, la mayoría de los trabajos epidemiológicos están llevados a cabo en relación a la salud dental o periodontal. En la actualidad los trabajos dedicados a estudiar a la patología de la mucosa oral siguen siendo escasos (1).

La prevalencia de las lesiones orales varia ampliamente entre el $16 \%$ y el $61 \%$, tanto en sujetos institucionalizados como no institucionalizados; estos amplios márgenes entre los distintos trabajos, hacen difícil la interpretación de los datos. Las diferencias encontradas en los diversos estudios pueden ser debidas a la distinta metodología utilizada, al tamaño y selección de la muestra, a la variabilidad interexaminador, experiencia del explorador así como al número de indicadores usados en su diagnostico (2-16).

Numerosos factores están implicados en esta patología oral como infecciones, traumatismos, agentes químicos, enfermedades sistémicas, cambios en el sistema inmunológico. Además de factores socioeconómicos, étnicos y ocupacionales (17-23).

El objetivo de este trabajo fue determinar la prevalencia de las lesiones mucosas siguiendo los criterios de la OMS en adultos mayores institucionalizados y no institucionalizados de 65 años o mas de la región de Murcia.

\section{MATERIAL Y MÉTODOS}

\section{Población objetivo}

Población mayor de 64 años residente en la Región de Murcia institucionalizada y no institucionalizada. El tamaño muestral se determinó separadamente para la población no institucionalizada y la institucionalizada. Para la población no institucionalizada se calculó el tamaño muestral para una prevalencia esperada de edentulismo del $50 \%$, con un error máximo admisible de $\pm 5 \%$ a un nivel de confianza del $95 \%$, y para una tasa de respuesta esperada del $70 \%$. Se estimó un tamaño muestral necesario de 545 personas. En la población institucionalizada, se determinó para una prevalencia esperada de edentulismo del $50 \%$, con un error máximo admisible de $\pm 6 \%$ a un nivel de confianza del $95 \%$. El tamaño muestral estimado fue de 245 sujetos.

\section{Procedimiento de muestreo}

Para la población no institucionalizada se realizó un muestreo aleatorio estratificado por sexo, edad y tipo de residencia (urbano/no urbano) proporcional a la distribución de la población a estudio. La extracción de la muestra se restringió al municipio de Murcia.

Para la selección de la parte urbana se incluyó a los individuos adscritos a todos los centros de salud pertenecientes al núcleo urbano del municipio en base a la Tarjeta Sanitaria. Para la extracción de los individuos correspondientes a la parte no urbana se seleccionó a los individuos adscritos a un centro de salud perteneciente a una entidad de población que cumplía los criterios de área no urbana y se realizó un muestro aleatorio estratificando por sexo y grupo de edad. El centro de salud seleccionado fue el de Patiño.

En el caso de los sujetos institucionalizados, se consideró a las residencias de personas mayores seleccionables como conglomerados. Se realizó una selección por conveniencia de 4 centros, en los cuales se estudió a todos los individuos elegibles. Las residencias seleccionas fueron San Basilio (Murcia), Virgen de la Fuensanta (Murcia), Virgen del Rosario (Alhama de Murcia) y Domingo Sastre Salas (Lorca). La autorización para la realización de este estudio fue obtenida de la dirección de cada uno de los centros.

Se consideraron como criterios de exclusión los siguientes:

- Fallecimiento.

- Enfermedad general grave que impidiera la realización de la exploración.

- Persona no localizable a través de los datos de la Tarjeta Sanitaria. Se sustituyeron los individuos que no se pudieron contactar, bien por errores en los datos personales disponibles, o bien por fracaso tras varios intentos de contacto. Para estos últimos se realizaron 5 intentos de contacto en diferentes franjas horarias antes de dar por ilocalizable al sujeto. Para los individuos de la 
muestra que presentaron alguno de los criterios de exclusión se seleccionaron aleatoriamente sustitutos de su mismo sexo, grupo de edad y pertenecientes a su mismo centro de salud. Para la muestra institucionalizada el único criterio de exclusión fue la existencia de enfermedad general grave que impidiera la exploración o no querer participar en el estudio.

Para la recogida de datos se han seguido las directrices y estrategias establecidas por la OMS en relación a las lesiones de los tejidos blandos bucales $(24,25)$. El tiempo empleado en la recogida de la información fue de 24 meses. A todos los pacientes se les realizó una exploración de su cavidad bucal. Dicha exploración se realizó en las dependencias del Centro de Salud y siempre por el mismo explorador. El instrumental clínico utilizado consistió en: espejos dentales planos (dos por cada exploración), sondas odontológicas, guantes, mascarillas. Todo el instrumental no desechable fue convenientemente esterilizado para su uso.

Los datos fueron introducidos en una base de datos y se empleo el SPSS11.5 Se realizó un análisis descriptivo de las variables. Las variables continuas se expresan en forma de media. Las proporciones se comparan mediante la prueba de $\chi 2$ de Pearson. Se efectuó la prueba de $\chi 2$ de tendencia lineal para valorar la prevalencia de lesiones en cada grupo de edad. La diferencia entre dos o más medias se determinó mediante la $t$ de Student y el análisis de la varianza. En todos los cálculos se aceptó un nivel de significación $\mathrm{p}<0,05$.

\section{RESULTADOS}

Se completaron un total de 385 exploraciones en población general, lo que supuso una tasa de respuestas del $46 \%$, siendo similar en la población urbana y rural $(45,5 \%$ y $50 \%$ respectivamente). Se encontraron diferencias estadísticamente significativas en la tasa respuesta por sexo y por grupos de edad. La tasa de respuesta en hombres fue del $56,2 \%$ frente al $38,6 \%$ de las mujeres $(p<0,001)$. En relación con la edad se observó una tendencia decreciente con el aumento de la edad, variando desde una tasa de respuesta del $61 \%$ en el grupo de 65-69 años hasta el $25 \%$ en los individuos de 80 y más años $(\mathrm{p}<0,001)$. En la población institucionalizada se superó en todos los centros una tasa de respuesta del 99\% entre los sujetos elegibles.

En el $80,2 \%$ de los casos de sujetos no institucionalizado el estado de la mucosa era normal y $85,8 \%$ de los institucionalizados (Tablas 1 a 5)

Las úlceras traumáticas fue la patología mas frecuente tanto en institucionalizados como no institucionalizados. Otras alteraciones encontradas fueron candidiasis oral y lengua geográfica. La localización mas frecuente de las lesiones fue en la lengua. No apareció en nuestra muestra ningún caso sospechoso de neoplasia oral.

Las mujeres presentan mayor de lesiones mucosas aunque no existen diferencias significativas. Los sujetos institucionalizados presentan menor número de lesiones aunque las diferencias son poco importantes. Se presentan un mayor número de lesiones mucosas entre los sujetos con prótesis aunque las diferencias no son relevantes.

\section{DISCUSIÓN}

En este trabajo se ha seguido como instrumento de medición los criterios de la OMS para la identificación de las lesiones orales; sin embargo, pensamos que esta clasificación es indefinida e implica una detección limitada a las entidades más importantes. Un grupo numeroso grupo de lesiones orales quedan englobadas en el cajón de sastre de "Otras patologías".

Nosotros encontramos una prevalencia de lesiones mucosas de $17,8 \%$ en sujetos no institucionalizados y de $14,2 \%$ en institucionalizados, no obteniendo un valor de significación estadística entre estos dos grupos de población $(p=0,110)$. Estos valores obtenidos son ligeramente menores que en otros trabajos, estas diferencias podrían ser debida a distintas causas ( metodología utilizada, experiencia del explorador etc) (16-19).

En nuestro estudio, no encontramos diferencias estadísticamente significativas en relación a las lesio- 
AVANCES EN ODONTOESTOMATOLOGÍA

Vol. 22 - Núm. 6 - 2006

TABLA 1.- LESIONES DE LA MUCOSA ORAL SEGÚN SEXO

Alteración de la mucosa oral

- Ningún estado anormal

- Tumor maligno

- Leucoplasia

- Liquen plano

- Úlcera

- Gingivitis necrotizante

- Candidiasis

- Absceso

- Lengua geográfica

- Otras

Localización del transtorno

- Borde bermellón

- Comisuras

- Labios

- Surcos

- Mucosa bucal

- Suelo de la boca

- Lengua

- Paladar

- Rebordes alveolares/encías

(*) Comparando "Ningún estado anormal" frente a "Algún transtorno" P valor 0,369.

\begin{tabular}{|rr|rr|rr|}
\hline \multicolumn{7}{|c|}{ Sexo } \\
\hline \multicolumn{2}{|c|}{ Hombres } & \multicolumn{2}{c|}{ Mujeres } & \multicolumn{2}{c|}{ Total } \\
$\mathbf{N}$ & $\%$ & N & $\%$ & \multicolumn{1}{c|}{$\%$} \\
180 & 84,5 & 205 & 81,3 & 385 & 82,8 \\
0 & 0,0 & 0 & 0,0 & 0 & 0,0 \\
1 & 0,5 & 1 & 0,4 & 2 & 0,4 \\
0 & 0,0 & 0 & 0,0 & 0 & 0,0 \\
10 & 4,7 & 20 & 7,9 & 30 & 6,5 \\
0 & 0,0 & 0 & 0,0 & 0 & 0,0 \\
5 & 2,3 & 3 & 1,2 & 8 & 1,7 \\
3 & 1,4 & 0 & 0,0 & 3 & 0,6 \\
5 & 2,3 & 7 & 2,8 & 12 & 2,6 \\
9 & 4,2 & 16 & 6,3 & 25 & 5,4 \\
& & & & & \\
0 & 0,0 & 0 & 0,0 & 0 & 0,0 \\
6 & 18,2 & 1 & 2,1 & 7 & 8,8 \\
6 & 18,2 & 2 & 4,3 & 8 & 10,0 \\
0 & 0,0 & 2 & 4,3 & 2 & 2,5 \\
2 & 6,1 & 7 & 14,9 & 9 & 11,3 \\
0 & 0,0 & 4 & 8,5 & 4 & 5,0 \\
6 & 18,2 & 17 & 36,2 & 23 & 28,8 \\
4 & 12,1 & 4 & 8,5 & 8 & 10,0 \\
9 & 27,3 & 10 & 21,3 & 19 & 23,8 \\
\hline
\end{tabular}

TABLA 2.- LESIONES DE LA MUCOSA ORAL SEGÚN GRUPO DE EDAD

\begin{tabular}{|c|c|c|c|c|c|c|c|c|}
\hline & \multicolumn{8}{|c|}{ Grupo de edad } \\
\hline & \multicolumn{2}{|c|}{$65-69$} & \multicolumn{2}{|c|}{$70-74$} & \multicolumn{2}{|c|}{$75-79$} & \multicolumn{2}{|c|}{80 y más } \\
\hline & $\mathrm{N}$ & $\%$ & $\mathrm{~N}$ & $\%$ & $\mathrm{~N}$ & $\%$ & $\mathbf{N}$ & $\%$ \\
\hline $\begin{array}{l}\text { Alteración de la mucosa oral } \\
\text { - Ningún estado anormal }\end{array}$ & 97 & 82,2 & 85 & 78,7 & 67 & 80,7 & 133 & 86,9 \\
\hline - Tumor maligno & 0 & 0,0 & 0 & 0,0 & 0 & 0,0 & 0 & 0,0 \\
\hline - Leucoplasia & 0 & 0,0 & 2 & 1,9 & 0 & 0,0 & 0 & 0,0 \\
\hline - Liquen plano & 0 & 0,0 & 0 & 0,0 & 0 & 0,0 & 0 & 0,0 \\
\hline - Úlcera & 4 & 3,4 & 9 & 8,3 & 6 & 7,2 & 11 & 7,2 \\
\hline - Gingivitis necrotizante & 0 & 0,0 & 0 & 0,0 & 0 & 0,0 & 0 & 0,0 \\
\hline - Candidiasis & 0 & 0,0 & 2 & 1,9 & 2 & 2,4 & 4 & 2,6 \\
\hline - Absceso & 3 & 2,5 & 0 & 0,0 & 0 & 0,0 & 0 & 0,0 \\
\hline - Lengua geográfica & 5 & 4,2 & 2 & 1,9 & 3 & 3,6 & 2 & 1,3 \\
\hline - Otras & 9 & 7,6 & 8 & 7,4 & 5 & 6,0 & 3 & 2,0 \\
\hline Localización del transtorno & & & & & & & & \\
\hline - Borde bermellón & 0 & 0,0 & 0 & 0,0 & 0 & 0,0 & 0 & 0,0 \\
\hline - Comisuras & 1 & 4,8 & 4 & 17,4 & 2 & 12,5 & 0 & 0,0 \\
\hline - Labios & 2 & 9,5 & 5 & 21,7 & 1 & 6,3 & 0 & 0,0 \\
\hline - Surcos & 0 & 0,0 & 0 & 0,0 & 1 & 6,3 & 1 & 5,0 \\
\hline - Mucosa bucal & 1 & 4,8 & 3 & 13,0 & 2 & 12,5 & 3 & 15,0 \\
\hline - Suelo de la boca & 2 & 9,5 & 0 & 0,0 & 0 & 0,0 & 2 & 10,0 \\
\hline - Lengua & 5 & 23,8 & 5 & 21,7 & 5 & 31,3 & 8 & 40,0 \\
\hline - Paladar & 4 & 19,0 & 3 & 13,0 & 0 & 0,0 & 1 & 5,0 \\
\hline - Rebordes alveolares/encías & 6 & 28,6 & 3 & 13,0 & 5 & 31,3 & 5 & 25,0 \\
\hline
\end{tabular}

(*) Comparando "Ningún estado anormal” frente a "Algún transtorno" P valor 0,339. 
TABLA 3.- LESIONES DE LA MUCOSA ORAL SEGÚN INSTITUCIONALIZACIÓN

\begin{tabular}{|c|c|c|c|c|}
\hline & & & & \\
\hline & No $\mathrm{i}$ & zados & Ins & dos \\
\hline & $\mathbf{N}$ & $\%$ & $\mathrm{~N}$ & $\%$ \\
\hline Trastorno de la mucosa oral & & & & \\
\hline & 203 & 80,2 & 182 & 85,8 \\
\hline - Tumor maligno & 0 & 0,0 & $\begin{array}{l}0 \\
1\end{array}$ & 0,0 \\
\hline $\begin{array}{l}\text { - Leucoplasia } \\
\text { - Liquen plano }\end{array}$ & $\begin{array}{l}1 \\
0\end{array}$ & $\begin{array}{l}0,4 \\
0,0\end{array}$ & 0 & $\begin{array}{l}0,5 \\
0,0\end{array}$ \\
\hline - Úlcera & 15 & 5,9 & 15 & 7,1 \\
\hline - Gingivitis necrotizante & 0 & 0,0 & 0 & 0,0 \\
\hline - Candidiasis & 3 & 1,2 & 5 & 2,4 \\
\hline - Absceso & 3 & 1,2 & 0 & 0,0 \\
\hline - Lengua geográfica & 9 & 3,6 & 3 & 1,4 \\
\hline - Otras & 19 & 7,5 & 6 & 2,8 \\
\hline Localización de la alteración & & & & \\
\hline - Borde bermellón & 0 & 0,0 & 0 & 0,0 \\
\hline - Comisuras & 5 & 10,0 & 2 & 6,7 \\
\hline - Labios & 7 & 14,0 & 1 & 3,3 \\
\hline - Surcos & 0 & 0,0 & 2 & 6,7 \\
\hline - Mucosa bucal & 5 & 10,0 & 4 & 13,3 \\
\hline - Suelo de la boca & 2 & 4,0 & 2 & 6,7 \\
\hline - Lengua & 13 & 26,0 & 10 & 33,3 \\
\hline - Paladar & 7 & 14,0 & 1 & 3,3 \\
\hline - Rebordes alveolares/encías & 11 & 22,0 & 8 & 26,7 \\
\hline
\end{tabular}

(*) Comparando "Ningún estado anormal” frente a "Algún transtorno" P valor 0,110.

\section{TABLA 4.- LESIONES DE LA MUCOSA ORAL SEGÚN NÚCLEO DE POBLACIÓN}

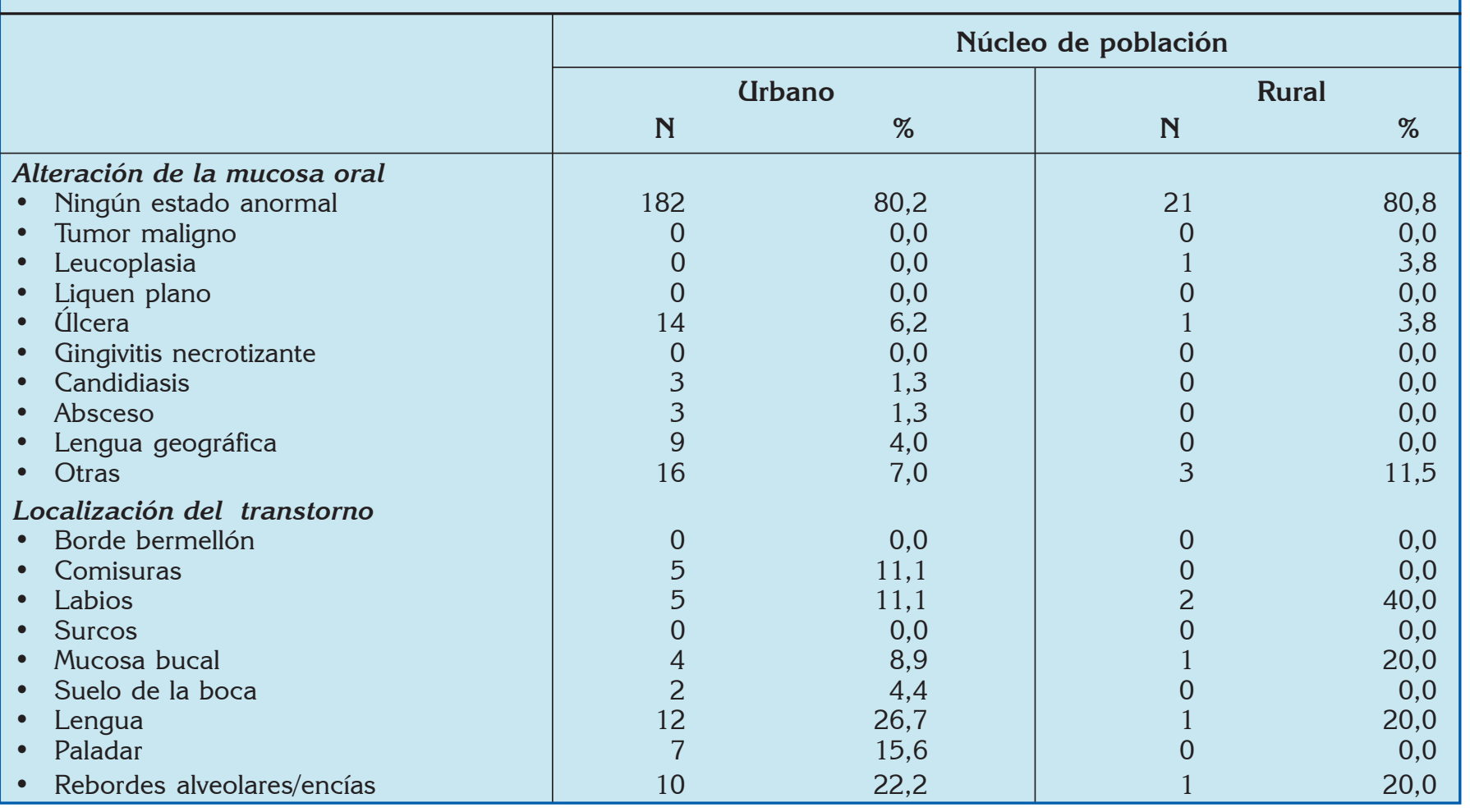

(*) Comparando "Ningún estado anormal" frente a "Algún transtorno" P valor 0,943. 
TABLA 5.- LESIONES DE LA MUCOSA ORAL SEGÚN NIVEL DE ESTUDIOS

\begin{tabular}{|c|c|c|c|c|c|c|c|c|c|c|c|c|}
\hline & \multicolumn{12}{|c|}{ Nivel de estudios } \\
\hline & \multicolumn{2}{|c|}{ Analfabeto } & \multicolumn{2}{|c|}{ Lee/escribe } & \multicolumn{2}{|c|}{ Primarios } & \multicolumn{2}{|c|}{ Secundarios } & \multicolumn{2}{|c|}{$\begin{array}{l}\text { Formación } \\
\text { profesional }\end{array}$} & \multicolumn{2}{|c|}{$\begin{array}{l}\text { Universi- } \\
\text { tarios }\end{array}$} \\
\hline & $N$ & $\%$ & $\mathrm{~N}$ & $\%$ & $\mathrm{~N}$ & $\%$ & $\mathrm{~N}$ & $\%$ & $N$ & $\%$ & $\mathrm{~N}$ & $\%$ \\
\hline $\begin{array}{l}\text { Alteración de la mucosa } \\
\text { oral }\end{array}$ & & & & & & & & & & & & \\
\hline - Ningún estado anormal & 25 & 86,2 & 130 & 80,7 & 165 & 82,9 & 27 & 93,1 & 12 & 80,0 & 26 & 81,3 \\
\hline - Tumor maligno & 0 & 0,0 & 0 & 0,0 & 0 & 0,0 & 0 & 0,0 & 0 & 0,0 & 0 & 0,0 \\
\hline - Leucoplasia & c & 0,0 & 1 & 0,6 & 0 & 0,0 & 1 & 3,4 & 0 & 0,0 & 0 & 0,0 \\
\hline - Liquen plano & 0 & 0,0 & 0 & 0,0 & 0 & 0,0 & 0 & 0,0 & 0 & 0,0 & 0 & 0,0 \\
\hline - Úlcera & 3 & 10,3 & 14 & 8,7 & 9 & 4,5 & 0 & 0,0 & 2 & 13,3 & 2 & 6,3 \\
\hline - Gingivitis necrotizante & $c$ & 0,0 & 0 & 0,0 & 0 & 0,0 & 0 & 0,0 & 0 & 0,0 & 0 & 0,0 \\
\hline - Candidiasis & c & 0,0 & 4 & 2,5 & 4 & 2,0 & 0 & 0,0 & 0 & 0,0 & 0 & 0,0 \\
\hline - Absceso & c & 0,0 & 0 & 0,0 & 1 & 0,5 & 0 & 0,0 & 1 & 6,7 & 1 & 3,1 \\
\hline - Lengua geográfica & $c$ & 0,0 & 3 & 1,9 & 8 & 4,0 & 0 & 0,0 & 0 & 0,0 & 1 & 3,1 \\
\hline - Otras & 1 & 3,4 & 9 & 5,6 & 12 & 6,0 & 1 & 3,4 & 0 & 0,0 & 2 & 6,3 \\
\hline Localización transtorno & c & 00 & 0 & 00 & 0 & 00 & 0 & 00 & 0 & 00 & 0 & 00 \\
\hline - Comisuras & 1 & 25,0 & 0 & 0,0 & 5 & 14,7 & 1 & 50,0 & 0 & 0,0 & 0 & 0,0 \\
\hline - Labios & 1 & 25,0 & 3 & 9,7 & 3 & 8,8 & 0 & 0,0 & 1 & 33,3 & 0 & 0,0 \\
\hline - Surcos & c & 0,0 & 2 & 6,5 & 0 & 0,0 & 0 & 0,0 & 0 & 0,0 & 0 & 0,0 \\
\hline - Mucosa bucal & 2 & 50,0 & 4 & 12,9 & 2 & 5,9 & 0 & 0,0 & 1 & 33,3 & 0 & 0,0 \\
\hline - Suelo de la boca & 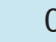 & 0,0 & 2 & 6,5 & 2 & 5,9 & 0 & 0,0 & 0 & 0,0 & 0 & 0,0 \\
\hline - Lengua & c & 0,0 & 7 & 22,6 & 13 & 38,2 & 1 & 50,0 & 0 & 0,0 & 2 & 33,3 \\
\hline - Paladar & $c$ & 0,0 & 3 & 9,7 & 3 & 8,8 & 0 & 0,0 & 0 & 0,0 & 2 & 33,3 \\
\hline - Rebordes alveolares/encías & c & 0,0 & 10 & 32,3 & 6 & 17,6 & 0 & 0,0 & 1 & 33,3 & 2 & 33,3 \\
\hline
\end{tabular}

(*) Comparando "Ningún estado anormal" frente a "Algún transtorno" P valor 0,69.

nes mucosa respecto al genero, ni tampoco si el paciente residía en área urbana o rural; o si el sujeto mayor vivía en comunidad o en institución. Respecto a los distintos grupos de edad; pudimos observar que conforme aumenta la edad, este es más susceptible de presentar lesiones orales; pero no tuvimos valores estadísticamente significativos.

Las ulceraciones de origen traumático es la patología mas frecuente encontrada por nosotros, generalmente, se asocia a la presencia de prótesis, que provoca lesiones de decúbito en la mucosa que la soporta; estas alteraciones brindan una excepcional puerta de entrada a la actuación de carcinógenos como el tabaco y el alcohol y otros agentes todavía desconocidos. En este sentido es fundamental el estudio de factores exógenos ya que la combinación de tabaco, alcohol y prótesis defectuosas contribuyen a incrementar la susceptibilidad de sufrir cambios patológicos en la mucosa oral. El efecto traumático sostenido de las prótesis sobre las mucosas es algo que puede ser detectado y es, por tanto, un riesgo susceptible de ser controlado $(9,11)$. Debe evitarse una actitud conservadora en este tipo de lesiones y realizar el diagnóstico diferencial con otras lesiones ulceradas (neoplasias, alteraciones dermatológicas o inmunológicas, farmacológicas, etc).

La candidiasis oral es la lesión infecciosa por excelencia en este grupo de edad y su aparición se relaciona con diversos factores facilitadores, entre estos se encuentran factores locales como tabaco, alcohol, higiene, presencia de prótesis, etc, y factores generales como xerostomía, diabetes, enfermedades neoplásicas, alteraciones inmunitarias, fármacos (sobre todo antibióticos y corticosteroides) (11-15).

Es importante resaltar que las lesiones en la mucosa oral suelen ser más frecuentes en ancianos con prótesis dentales antiguas y/o defectuosas. De esta forma, cuantas más prótesis existen en la boca, mayor es la probabilidad de sufrir alteraciones en la muco- 
sa bucal. Las prótesis en peor estado son causantes de un mayor número de patología. Nosotros encontramos la estomatitis protésica con una tasa del 1,2\% $\%$ en no institucionalizados y $2,4 \%$ en institucionalizados. Sin embargo para Espinoza et al (21) encuentran que esta patología supone el $22,3 \%$ siendo la mas común seguida de hiperplasia irritativa $(9,4 \%)$ y varicosidades (9\%). El estudio realizado en la Universidad del País Vasco (6), la candidiasis oral por prótesis supone 14,1 por ciento de ancianos afectados. Además, en el 12,1 por ciento de la población geriátrica se han encontrado varices; en el 3,3 por ciento tumores benignos; en el 2,8 por ciento leucoplasias y en el 1,1\% liquen plano bucal. En nuestro trabajo no encontramos ningún caso de lesión neoplásica y tampoco encontramos liquen plano oral. Si encontramos en dos casos lesiones compatibles de leucoplasia oral.

Entre los ancianos seria prioritario la puesta en marcha de campañas y programas que fomenten los hábitos orales saludables así como, implementar exámenes periódicos ya que contribuyen a la prevención de las lesiones de la mucosa oral.

\section{AGRADECIMIENTOS}

Este trabajo ha sido realizado con Beca de la Fundación Séneca

PRSAN/03/FS/03

\section{BIBLIOGRAFÍA}

1. Barmes DE. A global view o oral disease: today and tomorrow. Community Dent Oral Epidemiol 1999;27:2-7.

2. Fleishman R, Peles D. B, Pisanti, S. Oral mucosal lesions among elderly in Israel. J Dent Res 1985; 64:831-6.

3. Hand J S, Whitehill J. M.The prevalence of oral mucosal lesions in an elderly population. JADA 1986;113:73-6.

4. Jorge J, Almeida O P, Bozzo L, Scully C, Graner E.Oral mucosal health and disease in insti- tutionalized elderly in Brazil. Community Dent Oral Epidemiol 1991; 19:173-5.

5. Rueda A, Mosqueda A, Zimbrón A, Vergara G. Prevalencia de lesiones en mucosa oral en ancianos. Práctica Odontológica 1987; 8: 38-47.

6. Rodríguez Baciero G, Goirena de Gandairas FJ, Mallo Pérez. Patología de la mucosa bucal en los ancianos españoles. Ediciones Eguia, SL Bilbao, 1999.

7. Velasco E, Obando R, Vigo M, Martinez A,Bullón P. La valoración del estado mucosa oral en el paciente geriátrico. Avances en Odontoestomatologia 1995; 11:691-700.

8. Johnson N, Warnakulasuriya K, Partridge M, Langdon J. Oral cancer-a serious and growing problem. Ann R Coll Surg Engl 1995;77: 3212.

9. Hoad-Reddick G. Oral pathology and prosthesesare they related? Investigation in an elderly population. J Oral Rehabilit 1989;16:75-87.

10. Jorge J, Almeida O, Bozzo L, Scully C, Graner E. Oral mucosal health and diseases in institutionalized elderly in Brasil. Community Dent Oral Epidemiol 1991;19:173-5.

11. Moskona D, Kaplan I. Oral lesions in elderly denture wearers. Clin Prevent Dent 1992;14: 11-4.

12. Vigild M. Oral mucosal lesions among institutionalized elderly in Denmark. Community Dent Oral Epidemiol 1987;15:309-13.

13. Onofre $M$, Sposto $M$, Navarro $C$ et al. Potentially malignant oral lesions: discrepancies between clinical and histological diagnosis. Oral Diseases 1997;3:148-52.

14. Zain RB, Ikeda N, Gupta PC, Warnakulasuriya S, van Wyk CW, Shresth a P, Axell T.chewing habits: consensus from a workshop held in Kuala Lumpur, Malaysia, November 25-27, 1996. J Oral Pathol Med. 1999;28:1-4. 
15. Taiyeb Ali TB, Razak IA, Raja Latifah RJ, Zain RB. An epidemiological survey of oral mucosal lesions among elderly Malaysians. Gerodontology. 1995; 12:37-40.

16. García-Pola Vallejo MJ, Martínez Díaz-Canel AI, García Martín JM, González García M. Risk factors for oral soft tissue lesions in an adult Spanish population Community Dent Oral Epidemiol. 2002;30:277-85.

17. Mallo Pérez L, Rodríguez-Baciero G, Goirena de Gandarias FJ, Lafuente Urdinguio P. Patología de la mucosa oral en los ancianos institucionalizados españoles. Medicina Oral 2000;5:17786.

18. Subirá C, Precioso JL, Ramón JM, Valverde A. Prevalencia de patología oral entre los españoles de 65 o mas años. Archivos Odontoestomatol 2004;20:512-20

19. Jainkittivong A, Aneksuk V, Langlais RP. Oral mucosal conditions in elderly dental patients Oral Dis. 2002;8:218-23

20. Campisi G, Margiotta V. Oral mucosal lesions and risk habits among men in an Italian study population.J Oral Pathol Med. 2001;30:22-8.

21. Espinoza I, Rojas R, Aranda W, Gamonal J. Prevalence of oral mucosal lesions in elderly people in Santiago, Chile. J Oral Pathol Med. 2003;32:571-5.

22. Andreasen JO, Pindborg JJ, Hjorting-Hansen E, Axell $\mathrm{T}$ Oral health care: more than caries and periodontal disease. A survey of epidemiological studies on oral disease. Int Dent J. 1986;36: 207-14.

23. Reichart PA. Oral mucosal lesions in a representative cross-sectional study of aging Germans. Community Dent Oral Epidemiol. 2000;28:390-8.

24. WHO. Oral health surveys. Basic methods. 4th ed Geneve:WHO;1997.

25. WHO. Guide to epidemiology and diagnosis of oral mucosal diseases and conditions. Community Dent Oral Epidemiol 1980;8:1-26.

\section{CORRESPONDENCIA}

López Jornet $\mathrm{P}$

Clínica Odontológica Universitaria

Medicina Bucal Hospital Morales Meseguer

Adv. Marques de los Vélez, s/n

3008 Murcia

Tel.: 968230061

Fax: 968239565

Email: majornet@um.es 